Proc. Indian Acad. Sci. (Math. Sci.) Vol. 117, No. 3, August 2003, pp. 371-385.

Printed in India

\title{
Weighted composition operators from Bergman-type spaces into Bloch spaces
}

\author{
SONGXIAO LI ${ }^{1,2}$ and STEVO STEVIĆ 3 \\ ${ }^{1}$ Department of Mathematics, Shantou University, 515063 Shantou, Guangdong, China \\ ${ }^{2}$ Department of Mathematics, Jia Ying University, 514015 Meizhou, Guangdong, \\ China \\ ${ }^{3}$ Mathematical Institute of the Serbian Academy of Science, Knez Mihailova 35/I, \\ 11000 Beograd, Serbia \\ E-mail: jyulsx@163.com; 1sx@mail.zjxu.edu.cn; sstevic@ptt.yu; \\ sstevo@matf.bg.ac.yu \\ MS received 5 June 2006; revised 3 October 2006
}

Abstract. Let $\varphi$ be an analytic self-map and $u$ be a fixed analytic function on the open unit disk $D$ in the complex plane $\mathbb{C}$. The weighted composition operator is defined by

$$
u C_{\varphi} f=u \cdot(f \circ \varphi), f \in H(D) .
$$

Weighted composition operators from Bergman-type spaces into Bloch spaces and little Bloch spaces are characterized by function theoretic properties of their inducing maps.

Keywords. Weighted composition operator; Bergman-type space; Bloch space.

\section{Introduction}

Let $D$ be the open unit disk in the complex plane $\mathbb{C}$. Denote by $H(D)$ the class of all functions analytic on $D$. An analytic self-map $\varphi: D \rightarrow D$ induces the composition operator $C_{\varphi}$ on $H(D)$, defined by $C_{\varphi}(f)=f(\varphi(z))$ for $f$ analytic on $D$. It is a well-known consequence of Littlewood's subordination principle that the composition operator $C_{\varphi}$ is bounded on the classical Hardy and Bergman spaces (see, for example [1]).

Recall that a linear operator is said to be bounded if the image of a bounded set is a bounded set, while a linear operator is compact if it takes bounded sets to sets with compact closure. It is interesting to provide a function theoretic characterization of when $\varphi$ induces a bounded or compact composition operator on various spaces. The book [1] contains plenty of information on this topic.

Let $u$ be a fixed analytic function on the open unit disk. Define a linear operator $u C_{\varphi}$ on the space of analytic functions on $D$, called a weighted composition operator, by $u C_{\varphi} f=u \cdot(f \circ \varphi)$, where $f$ is an analytic function on $D$. We can regard this operator as a generalization of a multiplication operator and a composition operator.

A positive continuous function $\phi$ on $[0,1)$ is called normal, if there exist positive numbers $s$ and $t, 0<s<t$, such that

$$
\frac{\phi(r)}{(1-r)^{s}} \downarrow 0, \quad \frac{\phi(r)}{(1-r)^{t}} \uparrow \infty
$$

as $r \rightarrow 1^{-}$(see, for example [2|10]). 
For $0<p<\infty, 0<q<\infty$ and a normal function $\phi$, let $H(p, q, \phi)$ denote the space of all analytic functions $f$ on the unit disk $D$ such that

$$
\|f\|_{p, q, \phi}=\left(\int_{0}^{1} M_{q}^{p}(r, f) \frac{\phi^{p}(r)}{1-r} r \mathrm{~d} r\right)^{1 / p}<\infty
$$

where the integral means $M_{p}(f, r)$ are defined by

$$
M_{p}(f, r)=\left(\frac{1}{2 \pi} \int_{0}^{2 \pi}\left|f\left(r \mathrm{e}^{i \theta}\right)\right|^{p} \mathrm{~d} \theta\right)^{1 / p}, \quad 0 \leq r<1 .
$$

For $1 \leq p<\infty, H(p, q, \phi)$, equipped with the norm $\|\cdot\|_{p, q, \phi}$ is a Banach space. When $0<p<1,\|f\|_{p, q, \phi}$ is a quasinorm on $H(p, q, \phi), H(p, q, \phi)$ is a Frechet space but not a Banach space. If $0<p=q<\infty$, then $H(p, p, \phi)$ is the Bergman-type space

$$
H(p, p, \phi)=\left\{f \in H(D): \int_{D}|f(z)|^{p} \frac{\phi^{p}(|z|)}{1-|z|} \mathrm{d} A(z)<\infty\right\} .
$$

Here $\mathrm{d} A$ denotes the normalized Lebesgue area measure on the unit disk $D$ such that $A(D)=1$. Note that if $\phi(r)=(1-r)^{1 / p}$, then $H(p, p, \phi)$ is the Bergman space $A^{p}$.

An analytic function $f$ in $D$ is said to belong to the Bloch space $\mathscr{B}$ if

$$
B(f)=\sup _{z \in D}\left(1-|z|^{2}\right)\left|f^{\prime}(z)\right|<\infty .
$$

The expression $B(f)$ defines a seminorm while the natural norm is given by $\|f\|_{\mathscr{B}}=$ $|f(0)|+B(f)$. The norm makes $\mathscr{B}$ into a conformally invariant Banach space. Let $\mathscr{B}_{0}$ denote the subspace of $\mathscr{B}$ consisting of those $f \in \mathscr{B}$ for which $\left(1-|z|^{2}\right)\left|f^{\prime}(z)\right| \rightarrow 0$, as $|z| \rightarrow 1$. This space is called a little Bloch space. For more information on Bloch spaces see, for example [1/8|11|14|15] and the references therein.

In [5], Ohno has characterized the boundedness and compactness of weighted composition operators between $H^{\infty}$, the Bloch space $\mathscr{B}$ and the little Bloch space $\mathscr{B}_{0}$. In [7], Ohno and Zhao have characterized the boundedness and compactness of weighted composition operators on the Bloch space. Weighted composition operators between Blochtype spaces are characterized in [6] (see also [3]). In the setting of the unit ball or the unit polydisk, some necessary and sufficient conditions for a composition operator or weighted composition operator to be bounded or compact are given, for example, in [1]9[12]13].

In this paper we study the weighted composition operators from the Bergman-type space $H(p, p, \phi)$ into the Bloch space $\mathscr{B}$ and the little Bloch space $\mathscr{B}_{0}$. As corollaries, we obtain the complete characterizations of the boundedness and compactness of composition operators from Bergman spaces into Bloch spaces.

In this paper, constants are denoted by $C$, they are positive and may differ from one occurrence to the next. The notation $a \preceq b$ means that there is a positive constant $C$ such that $a \leq C b$. If both $a \preceq b$ and $b \preceq a$ hold, then one says that $a \asymp b$.

\section{Auxiliary results}

In this section, we give some auxiliary results which will be used in proving the main results of the paper. They are incorporated in the lemmas which follow. 
Lemma 2.1. Let $0<p<\infty$. If $f \in H(p, p, \phi)$, then

$$
|f(z)| \leq C \frac{\|f\|_{H(p, p, \phi)}}{\phi(|z|)\left(1-|z|^{2}\right)^{1 / p}}, \quad z \in D .
$$

Proof. Let $\beta(z, w)$ denote the Bergman metric between two points $z$ and $w$ in $D$. It is well-known that

$$
\beta(z, w)=\frac{1}{2} \log \frac{1+\left|\varphi_{z}(w)\right|}{1-\left|\varphi_{z}(w)\right|} .
$$

For $a \in D$ and $r>0$ the set $D(a, r)=\{z \in D: \beta(a, z)<r\}$ is the Bergman metric disk centered at $a$ with radius $r$. It is well-known that (see [14])

$$
\frac{\left(1-|a|^{2}\right)^{2}}{|1-\bar{a} z|^{4}} \asymp \frac{1}{\left(1-|z|^{2}\right)^{2}} \asymp \frac{1}{\left(1-|a|^{2}\right)^{2}} \asymp \frac{1}{|D(a, r)|},
$$

when $z \in D(a, r)$, where $|D(a, r)|$ denotes the area of the disk $D(a, r)$.

From (2) and since $\phi(r)$ is normal it is not difficult to see that for a fixed $r \in(0,1)$ the following relationship holds:

$$
\phi(|z|) \asymp \phi(|a|), \quad z \in D(a, r) .
$$

For $0<r<1$ and $z \in D$, by the subharmonicity of $|f(z)|^{p}$, (2) and (3), we have that

$$
\begin{aligned}
|f(z)|^{p} & \leq \frac{C}{\left(1-|z|^{2}\right)^{2}} \int_{D(z, r)}|f(w)|^{p} \mathrm{~d} A(w) \\
& \leq \frac{C}{\left(1-|z|^{2}\right) \phi^{p}(|z|)} \int_{D(z, r)}|f(w)|^{p} \frac{\phi^{p}(|w|)}{1-|w|} \mathrm{d} A(w) \\
& \leq \frac{C}{\left(1-|z|^{2}\right) \phi^{p}(|z|)} \int_{D}|f(w)|^{p} \frac{\phi^{p}(|w|)}{1-|w|} \mathrm{d} A(w) \\
& \leq \frac{C\|f\|_{H(p, p, \phi)}^{p}}{\left(1-|z|^{2}\right) \phi^{p}(|z|)}
\end{aligned}
$$

from which the desired result follows.

The following lemma can be found in [2]

Lemma 2.2. Let $0<p<\infty$. Then for $f \in H(D)$,

$$
\|f\|_{H(p, p, \phi)}^{p} \asymp|f(0)|^{p}+\int_{D}\left|f^{\prime}(z)\right|^{p}\left(1-|z|^{2}\right)^{p} \frac{\phi^{p}(|z|)}{1-|z|} \mathrm{d} A(z) .
$$

Lemma 2.3. Let $0<p<\infty$. If $f \in H(p, p, \phi)$ and $z \in D$, then

$$
\left|f^{\prime}(z)\right| \leq C \frac{\|f\|_{H(p, p, \phi)}}{\phi(|z|)\left(1-|z|^{2}\right)^{1 / p+1}}, \quad z \in D .
$$


Proof. By the subharmonicity of $\left|f^{\prime}(z)\right|^{p}$, (2) and (3), and Lemma 2.2 we have that

$$
\begin{aligned}
\left|f^{\prime}(z)\right|^{p} & \leq \frac{C}{\left(1-|z|^{2}\right)^{2}} \int_{D(z, r)}\left|f^{\prime}(w)\right|^{p} \mathrm{~d} A(w) \\
& \leq \frac{C}{\left(1-|z|^{2}\right)^{p+1} \phi^{p}(|z|)} \int_{D(z, r)} \frac{\phi^{p}(|w|)}{1-|w|}(1-|w|)^{p}\left|f^{\prime}(w)\right|^{p} \mathrm{~d} A(w) \\
& \leq \frac{C}{\left(1-|z|^{2}\right)^{p+1} \phi^{p}(|z|)} \int_{D} \frac{\phi^{p}(|w|)}{1-|w|}(1-|w|)^{p}\left|f^{\prime}(w)\right|^{p} \mathrm{~d} A(w) \\
& \leq \frac{C\|f\|_{H(p, p, \phi)}^{p}}{\left(1-|z|^{2}\right)^{p+1} \phi^{p}(|z|)},
\end{aligned}
$$

from which the result follows.

The following lemma can be found in [10].

Lemma 2.4. For $\beta>-1$ and $m>1+\beta$ we have

$$
\int_{0}^{1} \frac{(1-r)^{\beta}}{(1-\rho r)^{m}} \mathrm{~d} r \leq C(1-\rho)^{1+\beta-m}, \quad 0<\rho<1 .
$$

The following criterion for compactness follows by standard arguments similar, for example, to those outlined in Proposition 3.11 of [1].

Lemma 2.5. The operator $u C_{\varphi}: H(p, p, \phi) \rightarrow \mathscr{B}$ is compact if and only if for any bounded sequence $\left(f_{n}\right)_{n \in \mathbb{N}}$ in $H(p, p, \phi)$ which converges to zero uniformly on compact subsets of $D$, we have $\left\|u C_{\varphi} f_{n}\right\|_{\mathscr{B}} \rightarrow 0$ as $n \rightarrow \infty$.

\section{The boundedness and compactness of the operator $u C_{\varphi}: H(p, p, \phi) \rightarrow \mathscr{B}$}

In this section we characterize the boundedness and compactness of the weighted composition operator $u C_{\varphi}: H(p, p, \phi) \rightarrow \mathscr{B}$.

Theorem 3.1. Suppose that $\varphi$ is an analytic self-map of the unit disk, $u \in H(D), 0<p<$ $\infty$ and that $\phi$ is normal on $[0,1)$. Then, $u C_{\varphi}: H(p, p, \phi) \rightarrow \mathscr{B}$ is bounded if and only if the following conditions are satisfied:

$$
\sup _{z \in D} \frac{\left(1-|z|^{2}\right)\left|u^{\prime}(z)\right|}{\phi(|\varphi(z)|)\left(1-|\varphi(z)|^{2}\right)^{1 / p}}<\infty ;
$$

$$
\sup _{z \in D} \frac{\left(1-|z|^{2}\right)\left|u(z) \varphi^{\prime}(z)\right|}{\phi(|\varphi(z)|)\left(1-|\varphi(z)|^{2}\right)^{1+1 / p}}<\infty .
$$

Proof. Suppose that the conditions (i) and (ii) hold. For arbitrary $z$ in $D$ and $f \in$ $H(p, p, \phi)$, by Lemmas 2.1 and 2.3 we have 


$$
\begin{aligned}
\left(1-|z|^{2}\right)\left|\left(u C_{\varphi} f\right)^{\prime}(z)\right| & \\
\leq & \left(1-|z|^{2}\right)\left|u^{\prime}(z)\right||f(\varphi(z))|+\left(1-|z|^{2}\right)\left|f^{\prime}(\varphi(z))\right|\left|u(z) \varphi^{\prime}(z)\right| \\
\leq & \left(1-|z|^{2}\right)\left|u^{\prime}(z)\right| \frac{C\|f\|_{H(p, p, \phi)}}{\phi(|\varphi(z)|)\left(1-|\varphi(z)|^{2}\right)^{1 / p}} \\
& +C\left(1-|z|^{2}\right)\left|u(z) \varphi^{\prime}(z)\right| \frac{\|f\|_{H(p, p, \phi)}}{\phi(|\varphi(z)|)\left(1-|\varphi(z)|^{2}\right)^{1+1 / p}} \\
\leq & \left(\frac{C\left(1-|z|^{2}\right)\left|u^{\prime}(z)\right|}{\phi(|\varphi(z)|)\left(1-|\varphi(z)|^{2}\right)^{1 / p}}+\frac{C\left(1-|z|^{2}\right)\left|u(z) \varphi^{\prime}(z)\right|}{\phi(|\varphi(z)|)\left(1-|\varphi(z)|^{2}\right)^{1+1 / p}}\right)\|f\|_{H(p, p, \phi)} .
\end{aligned}
$$

Taking the supremum in (7) over $D$ and then using conditions (5) and (6) we obtain that the operator $u C_{\varphi}: H(p, p, \phi) \rightarrow \mathscr{B}$ is bounded.

Conversely, suppose that $u C_{\varphi}: H(p, p, \phi) \rightarrow \mathscr{B}$ is bounded. Then, taking the functions $f(z)=z$ and $f(z)=1$ we obtain that the quantities

$$
\sup _{z \in D}\left(1-|z|^{2}\right)\left|u(z) \varphi^{\prime}(z)+u^{\prime}(z) \varphi(z)\right| \quad \text { and } \quad \sup _{z \in D}\left(1-|z|^{2}\right)\left|u^{\prime}(z)\right|
$$

are finite. Using these facts and the boundedness of the function $\varphi(z)$, we have that

$$
\sup _{z \in D}\left(1-|z|^{2}\right)\left|u(z) \varphi^{\prime}(z)\right|<\infty .
$$

For fixed $w \in D$, take

$$
f_{w}(z)=\frac{\left(1-|w|^{2}\right)^{t+1}}{\phi(|w|)(1-\bar{w} z)^{1 / p+t+1}} .
$$

By Lemma 1.4 .10 of [8], we know that

$$
M_{p}\left(f_{w}, r\right) \leq C \frac{\left(1-|w|^{2}\right)^{t+1}}{\phi(|w|)(1-r|w|)^{t+1}} .
$$

Since $\phi$ is normal, by Lemma 2.4 , we obtain

$$
\begin{aligned}
\left\|f_{w}\right\|_{H(p, p, \phi)}^{p}= & \int_{0}^{1} M_{p}^{p}\left(f_{w}, r\right) \frac{\phi^{p}(r)}{1-r} r \mathrm{~d} r \\
\leq & C \int_{0}^{1} \frac{\left(1-|w|^{2}\right)^{p(t+1)}}{\phi^{p}(|w|)(1-r|w|)^{p(t+1)}} \frac{\phi^{p}(r)}{1-r} \mathrm{~d} r \\
\leq & C\left(\int_{0}^{|w|} \frac{\left(1-|w|^{2}\right)^{p(t+1)}}{\phi^{p}(|w|)(1-r|w|)^{p(t+1)}} \frac{\phi^{p}(r)}{1-r} \mathrm{~d} r\right. \\
& \left.+\int_{|w|}^{1} \frac{\left(1-|w|^{2}\right)^{p(t+1)}}{\phi^{p}(|w|)(1-r|w|)^{p(t+1)}} \frac{\phi^{p}(r)}{1-r} \mathrm{~d} r\right)
\end{aligned}
$$




$$
\begin{aligned}
\leq & C \frac{\left(1-|w|^{2}\right)^{p(t+1)}}{\phi^{p}(|w|)} \frac{\phi^{p}(|w|)}{\left(1-|w|^{2}\right)^{p t}} \int_{0}^{|w|} \frac{(1-r)^{p t-1}}{(1-r|w|)^{p(t+1)}} \mathrm{d} r \\
& +C \frac{\left(1-|w|^{2}\right)^{p(t+1)}}{\phi^{p}(|w|)} \frac{\phi^{p}(|w|)}{\left(1-|w|^{2}\right)^{p s}} \int_{|w|}^{1} \frac{(1-r)^{p s-1}}{(1-r|w|)^{p(t+1)}} d \leq C .
\end{aligned}
$$

Therefore $f_{w} \in H(p, p, \phi)$, and moreover $\sup _{w \in D}\left\|f_{w}\right\|_{H(p, p, \phi)} \leq C$. Hence, we have

$$
\begin{aligned}
C\left\|u C_{\varphi}\right\| & \geq\left\|f_{\varphi(\lambda)}\right\|_{H(p, p, \phi)}\left\|u C_{\varphi}\right\| \geq\left\|u C_{\varphi} f_{\varphi(\lambda)}\right\|_{\mathscr{B}} \\
& \geq\left|(1 / p+t+1) \frac{\left(1-|\lambda|^{2}\right)\left|u(\lambda) \overline{\varphi(\lambda)} \varphi^{\prime}(\lambda)\right|}{\phi(|\varphi(\lambda)|)\left(1-|\varphi(\lambda)|^{2}\right)^{1+1 / p}}-\frac{\left(1-|\lambda|^{2}\right)\left|u^{\prime}(\lambda)\right|}{\phi(|\varphi(\lambda)|)\left(1-|\varphi(\lambda)|^{2}\right)^{1 / p}}\right|,
\end{aligned}
$$

for every $\lambda \in D$, from which it follows that

$$
\begin{aligned}
& \frac{\left(1-|\lambda|^{2}\right)\left|u^{\prime}(\lambda)\right|}{\phi(|\varphi(\lambda)|)\left(1-|\varphi(\lambda)|^{2}\right)^{1 / p}} \\
& \quad \leq C\left\|u C_{\varphi}\right\|+(1 / p+t+1) \frac{\left(1-|\lambda|^{2}\right)\left|u(\lambda) \overline{\varphi(\lambda)} \varphi^{\prime}(\lambda)\right|}{\phi(|\varphi(\lambda)|)\left(1-|\varphi(\lambda)|^{2}\right)^{1+1 / p}} .
\end{aligned}
$$

Further, for $\lambda \in D$, take

$$
g_{\lambda}(z)=\frac{\left(1-|\varphi(\lambda)|^{2}\right)^{t+2}}{\phi(|\varphi(\lambda)|)(1-\overline{\varphi(\lambda)} z)^{1 / p+t+2}}-\frac{\left(1-|\varphi(\lambda)|^{2}\right)^{t+1}}{\phi(|\varphi(\lambda)|)(1-\overline{\varphi(\lambda)} z)^{1 / p+t+1}} .
$$

Then, $\sup _{\lambda \in D}\left\|g_{\lambda}\right\|_{H(p, p, \phi)} \leq C, g_{\lambda}(\varphi(\lambda))=0$ and

$$
g_{\lambda}^{\prime}(\varphi(\lambda))=\frac{\overline{\varphi(\lambda)}}{\phi(|\varphi(\lambda)|)\left(1-|\varphi(\lambda)|^{2}\right)^{1+1 / p}} .
$$

Thus,

$$
C\left\|u C_{\varphi}\right\| \geq\left\|u C_{\varphi} g_{\lambda}\right\|_{\mathscr{B}} \geq \frac{\left(1-|\lambda|^{2}\right)\left|u(\lambda) \overline{\varphi(\lambda)} \varphi^{\prime}(\lambda)\right|}{\phi(|\varphi(\lambda)|)\left(1-|\varphi(\lambda)|^{2}\right)^{1+1 / p}},
$$

i.e. we have

$$
\sup _{\lambda \in D} \frac{\left(1-|\lambda|^{2}\right)\left|u(\lambda) \overline{\varphi(\lambda)} \varphi^{\prime}(\lambda)\right|}{\phi(|\varphi(\lambda)|)\left(1-|\varphi(\lambda)|^{2}\right)^{1+1 / p}}<\infty .
$$

Thus for a fixed $\delta, 0<\delta<1$, by (11),

$$
\sup _{|\varphi(\lambda)|>\delta} \frac{\left(1-|\lambda|^{2}\right)|u(\lambda)|\left|\varphi^{\prime}(\lambda)\right|}{\phi(|\varphi(\lambda)|)\left(1-|\varphi(\lambda)|^{2}\right)^{1+1 / p}}<\infty .
$$

For $\lambda \in D$ such that $|\varphi(\lambda)| \leq \delta$, since $\phi$ is normal, we have

$$
\frac{\left(1-|\lambda|^{2}\right)\left|u(\lambda) \varphi^{\prime}(\lambda)\right|}{\phi(|\varphi(\lambda)|)\left(1-|\varphi(\lambda)|^{2}\right)^{1+1 / p}} \leq \frac{C}{\left(1-\delta^{2}\right)^{1+1 / p} \phi(\delta)}\left(1-|\lambda|^{2}\right)\left|u(\lambda) \varphi^{\prime}(\lambda)\right| .
$$


Hence, from (8) and (13), we obtain

$$
\sup _{|\varphi(\lambda)| \leq \delta} \frac{\left(1-|\lambda|^{2}\right)\left|u(\lambda) \varphi^{\prime}(\lambda)\right|}{\phi(|\varphi(\lambda)|)\left(1-|\varphi(\lambda)|^{2}\right)^{1+1 / p}}<\infty .
$$

The inequality in (6) follows from (12) and (14). Taking the supremum in (10) over $\lambda \in D$ and using (6), (5) follows. This completes the proof of the theorem.

Theorem 3.2. Suppose that $\varphi$ is an analytic self-map of the unit disk, $u \in H(D)$, $0<p<\infty$, that $\phi$ is normal on $[0,1)$ and that $u C_{\varphi}: H(p, p, \phi) \rightarrow \mathscr{B}$ is bounded. Then, $u C_{\varphi}: H(p, p, \phi) \rightarrow \mathscr{B}$ is compact if and only if the following conditions are satisfied:

$$
\lim _{|\varphi(z)| \rightarrow 1} \frac{\left(1-|z|^{2}\right)\left|u^{\prime}(z)\right|}{\phi(|\varphi(z)|)\left(1-|\varphi(z)|^{2}\right)^{1 / p}}=0
$$

$$
\lim _{|\varphi(z)| \rightarrow 1} \frac{\left(1-|z|^{2}\right)\left|u(z) \varphi^{\prime}(z)\right|}{\phi(|\varphi(z)|)\left(1-|\varphi(z)|^{2}\right)^{1+1 / p}}=0 .
$$

Proof. First assume that conditions (i) and (ii) hold. In order to prove that $u C_{\varphi}$ is compact, according to Lemma 2.5, it suffices to show that if $\left(f_{n}\right)_{n \in \mathbb{N}}$ is a bounded sequence in $H(p, p, \phi)$ that converges to 0 uniformly on compact subsets of $D$, then $\left\|u C_{\varphi} f_{n}\right\|_{\mathscr{B}} \rightarrow 0$ as $n \rightarrow \infty$. Let $\left(f_{n}\right)_{n \in \mathbb{N}}$ be a sequence in $H(p, p, \phi)$ with $\sup _{n \in \mathbb{N}}\left\|f_{n}\right\|_{H(p, p, \phi)} \leq L$ and suppose $f_{n} \rightarrow 0$ uniformly on compact subsets of $D$ as $n \rightarrow \infty$.

By the assumptions of the theorem we have that for any $\varepsilon>0$, there is a constant $\delta, 0<\delta<1$, such that $\delta<|\varphi(z)|<1$ implies

$$
\frac{\left(1-|z|^{2}\right)\left|u^{\prime}(z)\right|}{\phi(|\varphi(z)|)\left(1-|\varphi(z)|^{2}\right)^{1 / p}}<\varepsilon / L
$$

and

$$
\frac{\left(1-|z|^{2}\right)\left|u(z) \varphi^{\prime}(z)\right|}{\phi(|\varphi(z)|)\left(1-|\varphi(z)|^{2}\right)^{1+1 / p}}<\varepsilon / L .
$$

Let $K=\{w \in D:|w| \leq \delta\}$. Note that $K$ is a compact subset of $D$. From this, since $\phi$ is normal and using estimates from Lemmas 2.1 and 2.3, we have that

$$
\begin{aligned}
& \left\|u C_{\varphi} f_{n}\right\|_{\mathscr{B}} \\
& =\sup _{z \in D}\left(1-|z|^{2}\right)\left|\left(u C_{\varphi} f_{n}\right)^{\prime}(z)\right|+\left|u(0) f_{n}(\varphi(0))\right| \\
& \leq \sup _{z \in D}\left(1-|z|^{2}\right)\left|u^{\prime}(z) f_{n}(\varphi(z))\right|+\sup _{z \in D}\left(1-|z|^{2}\right)\left|u(z) f_{n}^{\prime}(\varphi(z)) \varphi^{\prime}(z)\right|+\left|u(0) f_{n}(\varphi(0))\right| \\
& \leq \sup _{\{z \in D: \varphi(z) \in K\}}\left(1-|z|^{2}\right)\left|u^{\prime}(z) f_{n}(\varphi(z))\right|+\sup _{\{z \in D: \delta \leq|\varphi(z)|<1\}}\left(1-|z|^{2}\right)\left|u^{\prime}(z) f_{n}(\varphi(z))\right| \\
& \quad+\sup _{\{z \in D: \varphi(z) \in K\}}\left(1-|z|^{2}\right)\left|u(z) \varphi^{\prime}(z)\right|\left|f_{n}^{\prime}(\varphi(z))\right|
\end{aligned}
$$




$$
\begin{aligned}
& +\sup _{\{z \in D:} \operatorname{sul}_{\delta \leq(z) \mid<1\}}\left(1-|z|^{2}\right)\left|u(z) \varphi^{\prime}(z)\right|\left|f_{n}^{\prime}(\varphi(z))\right|+\left|u(0) f_{n}(\varphi(0))\right| \\
& \leq\|u\|_{\mathscr{B}} \sup _{w \in K}\left|f_{n}(w)\right|+C \sup _{\{z \in D: \delta \leq|\varphi(z)|<1\}} \frac{\left(1-|z|^{2}\right)\left|u^{\prime}(z)\right|}{\phi(|\varphi(z)|)\left(1-|\varphi(z)|^{2}\right)^{1 / p}}\left\|f_{n}\right\|_{H(p, p, \phi)} \\
& \quad+M \sup _{w \in K}\left|f_{n}^{\prime}(w)\right|+C \sup _{\{z \in D: \delta \leq|\varphi(z)|<1\}} \frac{\left(1-|z|^{2}\right)\left|u(z) \varphi^{\prime}(z)\right|}{\phi(|\varphi(z)|)\left(1-|\varphi(z)|^{2}\right)^{1+1 / p}}\left\|f_{n}\right\|_{H(p, p, \phi)} \\
& \quad+\left|u(0) f_{n}(\varphi(0))\right| \\
& \leq\|u\|_{\mathscr{B}} \sup _{w \in K}\left|f_{n}(w)\right|+C \varepsilon+M \sup _{w \in K}\left|f_{n}^{\prime}(w)\right|+C \varepsilon+\left|u(0) f_{n}(\varphi(0))\right|,
\end{aligned}
$$

where we have used the fact that $u \in \mathscr{B}$ (see the proof of Theorem 3.1) and where

$$
M=\sup _{z \in D}\left(1-|z|^{2}\right)\left|u(z) \varphi^{\prime}(z)\right| .
$$

Since $K$ is compact and $\varphi \in H(D)$, it follows that, $\lim _{n \rightarrow \infty} \sup _{w \in K}\left|f_{n}(w)\right|=0$. The set $\{\varphi(0)\}$ is also compact so that $\lim _{n \rightarrow \infty}\left|u(0) f_{n}(\varphi(0))\right|=0$. By Cauchy's estimate, if $f_{n}$ is a sequence which converges on compacta of $D$ to zero, then the sequence $f_{n}^{\prime}$ also converges on compacta of $D$ to zero as $n \rightarrow \infty$. Employing these facts and letting $n \rightarrow \infty$ in the last inequality, we obtain that

$$
\limsup _{n \rightarrow \infty}\left\|u C_{\varphi} f_{n}\right\|_{\mathscr{B}} \leq 2 C \varepsilon .
$$

Since $\varepsilon$ is an arbitrary positive number it follows that the last limit is equal to zero. Therefore, $u C_{\varphi}: H(p, p, \phi) \rightarrow \mathscr{B}$ is compact.

Conversely, suppose $u C_{\varphi}: H(p, p, \phi) \rightarrow \mathscr{B}$ is compact. Let $\left(z_{n}\right)_{n \in \mathbb{N}}$ be a sequence in $D$ such that $\left|\varphi\left(z_{n}\right)\right| \rightarrow 1$ as $n \rightarrow \infty$. If such a sequence does not exist conditions (15) and (16) are automatically satisfied. Choose

$$
f_{n}(z)=\frac{\left(1-\left|\varphi\left(z_{n}\right)\right|^{2}\right)^{t+1}}{\phi\left(\left|\varphi\left(z_{n}\right)\right|\right)\left(1-\overline{\varphi\left(z_{n}\right)} z\right)^{1 / p+t+1}}, \quad n \in \mathbb{N} .
$$

Then, as above $\sup _{n \in \mathbb{N}}\left\|f_{n}\right\|_{H(p, p, \phi)} \leq C$ and $f_{n}$ converges to 0 uniformly on compact subsets of $D$ as $n \rightarrow \infty$. Since $u C_{\varphi}$ is compact, we have

$$
\left\|u C_{\varphi} f_{n}\right\|_{\mathscr{B}} \rightarrow 0 \quad \text { as } n \rightarrow \infty .
$$

Thus

$$
\begin{aligned}
& \left\|u C_{\varphi} f_{n}\right\|_{\mathscr{B}} \\
& \quad \geq \sup _{z \in D}\left(1-|z|^{2}\right)\left|\left(u C_{\varphi} f_{n}\right)^{\prime}(z)\right| \\
& \quad \geq\left|(1 / p+t+1) \frac{\left(1-\left|z_{n}\right|^{2}\right)\left|u\left(z_{n}\right) \overline{\varphi\left(z_{n}\right)} \varphi^{\prime}\left(z_{n}\right)\right|}{\phi\left(\left|\varphi\left(z_{n}\right)\right|\right)\left(1-\left|\varphi\left(z_{n}\right)\right|^{2}\right)^{1+1 / p}}-\frac{\left(1-\left|z_{n}\right|^{2}\right)\left|u^{\prime}\left(z_{n}\right)\right|}{\phi\left(\left|\varphi\left(z_{n}\right)\right|\right)\left(1-\left|\varphi\left(z_{n}\right)\right|^{2}\right)^{1 / p}}\right| .
\end{aligned}
$$


Hence, we obtain

$$
\begin{aligned}
& \lim _{\left|\varphi\left(z_{n}\right)\right| \rightarrow 1} \frac{(1 / p+t+1)\left(1-\left|z_{n}\right|^{2}\right)\left|u\left(z_{n}\right) \overline{\varphi\left(z_{n}\right)} \varphi^{\prime}\left(z_{n}\right)\right|}{\phi\left(\left|\varphi\left(z_{n}\right)\right|\right)\left(1-\left|\varphi\left(z_{n}\right)\right|^{2}\right)^{1+1 / p}} \\
& \quad=\lim _{\left|\varphi\left(z_{n}\right)\right| \rightarrow 1} \frac{\left(1-\left|z_{n}\right|^{2}\right)\left|u^{\prime}\left(z_{n}\right)\right|}{\phi\left(\left|\varphi\left(z_{n}\right)\right|\right)\left(1-\left|\varphi\left(z_{n}\right)\right|^{2}\right)^{1 / p}},
\end{aligned}
$$

if one of these two limits exists.

Next, let

$$
g_{n}(z)=\frac{\left(1-\left|\varphi\left(z_{n}\right)\right|^{2}\right)^{t+2}}{\phi\left(\left|\varphi\left(z_{n}\right)\right|\right)\left(1-\overline{\varphi\left(z_{n}\right)} z\right)^{1 / p+t+2}}-\frac{\left(1-\left|\varphi\left(z_{n}\right)\right|^{2}\right)^{t+1}}{\phi\left(\left|\varphi\left(z_{n}\right)\right|\right)\left(1-\overline{\varphi\left(z_{n}\right)} z\right)^{1 / p+t+1}}
$$

for a sequence $\left(z_{n}\right)_{n \in \mathbb{N}}$ in $D$ such that $\left|\varphi\left(z_{n}\right)\right| \rightarrow 1$ as $n \rightarrow \infty$. Then, $\left(g_{n}\right)_{n \in \mathbb{N}}$ is a bounded sequence in $H(p, p, \phi), g_{n} \rightarrow 0$ uniformly on every compact subset of $D$ as $n \rightarrow \infty$, $g_{n}\left(\varphi\left(z_{n}\right)\right)=0$ and

$$
g_{n}^{\prime}\left(\varphi\left(z_{n}\right)\right)=\frac{\overline{\varphi\left(z_{n}\right)}}{\phi\left(\left|\varphi\left(z_{n}\right)\right|\right)\left(1-\left|\varphi\left(z_{n}\right)\right|^{2}\right)^{1+1 / p}}
$$

Then

$$
\frac{\left(1-\left|z_{n}\right|^{2}\right)\left|u\left(z_{n}\right) \overline{\varphi\left(z_{n}\right)} \varphi^{\prime}\left(z_{n}\right)\right|}{\phi\left(\left|\varphi\left(z_{n}\right)\right|\right)\left(1-\left|\varphi\left(z_{n}\right)\right|^{2}\right)^{1+\frac{1}{p}}} \leq\left\|u C_{\varphi} g_{n}\right\|_{\mathscr{B}} \rightarrow 0
$$

as $n \rightarrow \infty$.

From (20) it follows that

$$
\lim _{|\varphi(z)| \rightarrow 1} \frac{\left(1-|z|^{2}\right)\left|u(z) \varphi^{\prime}(z)\right|}{\phi(|\varphi(z)|)\left(1-|\varphi(z)|^{2}\right)^{1+1 / p}}=0 .
$$

Therefore by (18), we have

$$
\lim _{|\varphi(z)| \rightarrow 1} \frac{\left(1-|z|^{2}\right)\left|u^{\prime}(z)\right|}{\phi(|\varphi(z)|)\left(1-|\varphi(z)|^{2}\right)^{1 / p}}=0 .
$$

From the last two theorems, we can easily obtain the following corollaries:

\section{COROLLARY 3.3.}

Suppose that $\varphi$ is an analytic self-map of the unit disk, $0<p<\infty$ and that $\phi$ is normal on $[0,1)$. Then, the composition operator $C_{\varphi}: H(p, p, \phi) \rightarrow \mathscr{B}$ is bounded if and only if the following condition is satisfied:

$$
\sup _{z \in D} \frac{\left(1-|z|^{2}\right)\left|\varphi^{\prime}(z)\right|}{\phi(|\varphi(z)|)\left(1-|\varphi(z)|^{2}\right)^{1+1 / p}}<\infty
$$




\section{COROLLARY 3.4}

Suppose that $\varphi$ is an analytic self-map of the unit disk, $0<p<\infty$, that $\phi$ is normal on $[0,1)$ and that $C_{\varphi}: H(p, p, \phi) \rightarrow \mathscr{B}$ is bounded. Then, $C_{\varphi}: H(p, p, \phi) \rightarrow \mathscr{B}$ is compact if and only if the following condition is satisfied:

$$
\lim _{|\varphi(z)| \rightarrow 1} \frac{\left(1-|z|^{2}\right)\left|\varphi^{\prime}(z)\right|}{\phi(|\varphi(z)|)\left(1-|\varphi(z)|^{2}\right)^{1+1 / p}}=0 .
$$

Since the Bergman space is a special case of $H(p, p, \phi)$, we have the following corollaries.

\section{COROLLARY 3.5.}

Suppose that $\varphi$ is an analytic self-map of the unit disk, $u \in H(D)$ and $0<p<\infty$. Then, $u C_{\varphi}: A^{p} \rightarrow \mathscr{B}$ is bounded if and only if the following conditions are satisfied:

$$
\sup _{z \in D} \frac{\left(1-|z|^{2}\right)\left|u^{\prime}(z)\right|}{\left(1-|\varphi(z)|^{2}\right)^{2 / p}}<\infty \quad \text { and } \quad \sup _{z \in D} \frac{\left(1-|z|^{2}\right)\left|u(z) \varphi^{\prime}(z)\right|}{\left(1-|\varphi(z)|^{2}\right)^{1+2 / p}}<\infty .
$$

\section{COROLLARY 3.6.}

Suppose that $\varphi$ is an analytic self-map of the unit disk, $u \in H(D), 0<p<\infty$ and that $u C_{\varphi}: A^{p} \rightarrow \mathscr{B}$ is bounded. Then, $u C_{\varphi}: A^{p} \rightarrow \mathscr{B}$ is compact if and only if the following conditions are satisfied:

$$
\lim _{|\varphi(z)| \rightarrow 1} \frac{\left(1-|z|^{2}\right)\left|u^{\prime}(z)\right|}{\left(1-|\varphi(z)|^{2}\right)^{2 / p}}=0 \quad \text { and } \quad \lim _{|\varphi(z)| \rightarrow 1} \frac{\left(1-|z|^{2}\right)\left|u(z) \varphi^{\prime}(z)\right|}{\left(1-|\varphi(z)|^{2}\right)^{1+2 / p}}=0 .
$$

\section{COROLLARY 3.7.}

Suppose that $\varphi$ is an analytic self-map of the unit disk and $0<p<\infty$. Then, $C_{\varphi}$ : $A^{p} \rightarrow \mathscr{B}$ is bounded if and only if the following condition is satisfied:

$$
\sup _{z \in D} \frac{\left(1-|z|^{2}\right)\left|\varphi^{\prime}(z)\right|}{\left(1-|\varphi(z)|^{2}\right)^{1+2 / p}}<\infty .
$$

\section{COROLLARY 3.8.}

Suppose that $\varphi$ is an analytic self-map of the unit disk, $0<p<\infty$ and that $C_{\varphi}: A^{p} \rightarrow$ $\mathscr{B}$ is bounded. Then, $C_{\varphi}: A^{p} \rightarrow \mathscr{B}$ is compact if and only if the following condition is satisfied:

$$
\lim _{|\varphi(z)| \rightarrow 1} \frac{\left(1-|z|^{2}\right)\left|\varphi^{\prime}(z)\right|}{\left(1-|\varphi(z)|^{2}\right)^{1+2 / p}}=0
$$

\section{The boundedness and compactness of the operator $u C_{\varphi}: H(p, p, \phi) \rightarrow \mathscr{B}_{0}$}

Next we characterize the boundedness and compactness of the weighted composition operators $u C_{\varphi}: H(p, p, \phi) \rightarrow \mathscr{B}_{0}$. For this purpose, we need the following lemmas. The first lemma can be found in [4]. 
Lemma 4.1. A closed set $K$ in $\mathscr{B}_{0}$ is compact if and only if it is bounded and satisfies

$$
\lim _{|z| \rightarrow 1} \sup _{f \in K}\left(1-|z|^{2}\right)\left|f^{\prime}(z)\right|=0 \text {. }
$$

Lemma 4.2. Suppose that $\varphi$ is an analytic self-map of the unit disk, $u \in H(D), 0<p<\infty$ and that $\phi$ is normal on $[0,1)$. Then,

$$
\lim _{|z| \rightarrow 1} \frac{\left(1-|z|^{2}\right)\left|u^{\prime}(z)\right|}{\phi(|\varphi(z)|)\left(1-|\varphi(z)|^{2}\right)^{1 / p}}=0
$$

if and only if

$$
\lim _{|\varphi(z)| \rightarrow 1} \frac{\left(1-|z|^{2}\right)\left|u^{\prime}(z)\right|}{\phi(|\varphi(z)|)\left(1-|\varphi(z)|^{2}\right)^{1 / p}}=0
$$

and

$$
u \in \mathscr{B}_{0}
$$

Proof. Suppose that (22) holds. Then

$$
\left(1-|z|^{2}\right)\left|u^{\prime}(z)\right| \leq \frac{C\left(1-|z|^{2}\right)\left|u^{\prime}(z)\right|}{\phi(|\varphi(z)|)\left(1-|\varphi(z)|^{2}\right)^{1 / p}} \rightarrow 0
$$

as $|z| \rightarrow 1$

If $|\varphi(z)| \rightarrow 1$, then $|z| \rightarrow 1$, from which it follows that

$$
\lim _{|\varphi(z)| \rightarrow 1} \frac{\left(1-|z|^{2}\right)\left|u^{\prime}(z)\right|}{\phi(|\varphi(z)|)\left(1-|\varphi(z)|^{2}\right)^{1 / p}}=0 .
$$

Conversely, suppose that (23) and (24) hold. By (23), for every $\varepsilon>0$, there exists $r \in(0,1)$,

$$
\frac{\left(1-|z|^{2}\right)\left|u^{\prime}(z)\right|}{\phi(|\varphi(z)|)\left(1-|\varphi(z)|^{2}\right)^{1 / p}}<\varepsilon
$$

when $r<|\varphi(z)|<1$. By (24), there exists $\sigma \in(0,1)$,

$$
\left(1-|z|^{2}\right)\left|u^{\prime}(z)\right| \leq \varepsilon\left(1-r^{2}\right)^{1 / p} \phi(r)
$$

when $\sigma<|z|<1$.

Therefore, when $\sigma<|z|<1$ and $r<|\varphi(z)|<1$, we have that

$$
\frac{\left(1-|z|^{2}\right)\left|u^{\prime}(z)\right|}{\phi(|\varphi(z)|)\left(1-|\varphi(z)|^{2}\right)^{1 / p}}<\varepsilon .
$$

If $|\varphi(z)| \leq r$ and $\sigma<|z|<1$, then since $\phi$ is normal, we obtain

$$
\frac{\left(1-|z|^{2}\right)\left|u^{\prime}(z)\right|}{\phi(|\varphi(z)|)\left(1-|\varphi(z)|^{2}\right)^{1 / p}}<\frac{(1-r)^{s}\left(1-|z|^{2}\right)\left|u^{\prime}(z)\right|}{\phi(r)\left(1-|\varphi(z)|^{2}\right)^{1 / p+s}}<\varepsilon .
$$

Combining (25) with (26), we obtain the desired result.

Similarly to the proof of the above lemma, we have the following. 
Lemma 4.3. Suppose that $\varphi$ is an analytic self-map of the unit disk, $u \in H(D), 0<p<\infty$ and that $\phi$ is normal on $[0,1)$. Then,

$$
\lim _{|z| \rightarrow 1} \frac{\left(1-|z|^{2}\right)\left|u(z) \varphi^{\prime}(z)\right|}{\phi(|\varphi(z)|)\left(1-|\varphi(z)|^{2}\right)^{1+1 / p}}=0
$$

if and only if

$$
\lim _{|\varphi(z)| \rightarrow 1} \frac{\left(1-|z|^{2}\right)\left|u(z) \varphi^{\prime}(z)\right|}{\phi(|\varphi(z)|)\left(1-|\varphi(z)|^{2}\right)^{1+1 / p}}=0
$$

and

$$
\lim _{|z| \rightarrow 1}(1-|z|)^{2}\left|u(z) \varphi^{\prime}(z)\right|=0 .
$$

Theorem 4.4. Suppose that $\varphi$ is an analytic self-map of the unit disk, $u \in H(D), 0<p<$ $\infty$ and that $\phi$ is normal on $[0,1)$. Then, $u C_{\varphi}: H(p, p, \phi) \rightarrow \mathscr{B}_{0}$ is bounded if and only if $u C_{\varphi}: H(p, p, \phi) \rightarrow \mathscr{B}$ is bounded, $u \in \mathscr{B}_{0}$ and

$$
\lim _{|z| \rightarrow 1}\left(1-|z|^{2}\right)\left|u(z) \varphi^{\prime}(z)\right|=0 .
$$

Proof. First assume that $u C_{\varphi}: H(p, p, \phi) \rightarrow \mathscr{B}_{0}$ is bounded. Then, it is clear that $u C_{\varphi}: H(p, p, \phi) \rightarrow \mathscr{B}$ is bounded. Taking the functions $f(z)=1$ and $f(z)=z$, we obtain that $u \in \mathscr{B}_{0}$ and $\lim _{|z| \rightarrow 1}\left(1-|z|^{2}\right)\left|u(z) \varphi^{\prime}(z)\right|=0$.

Conversely, assume that $u C_{\varphi}: H(p, p, \phi) \rightarrow \mathscr{B}$ is bounded, $u \in \mathscr{B}_{0}$ and $\lim _{|z| \rightarrow 1}(1-$ $\left.|z|^{2}\right)\left|u(z) \varphi^{\prime}(z)\right|=0$. Then, for each polynomial $p(z)$, we have that

$$
\begin{aligned}
& \left(1-|z|^{2}\right)\left|\left(u C_{\varphi} p\right)^{\prime}(z)\right| \\
& \quad \leq\left(1-|z|^{2}\right)\left|u^{\prime}(z)\right||p(\varphi(z))|+\left(1-|z|^{2}\right)\left|u(z) \varphi^{\prime}(z) p^{\prime}(\varphi(z))\right|,
\end{aligned}
$$

from which it follows that $u C_{\varphi} p \in \mathscr{B}_{0}$. Since the set of all polynomials is dense in $H(p, p, \phi)$, we have that for every $f \in H(p, p, \phi)$ there is a sequence of polynomials $\left(p_{n}\right)_{n \in \mathbb{N}}$ such that $\left\|f-p_{n}\right\|_{H(p, p, \phi)} \rightarrow 0$, as $n \rightarrow \infty$. Hence

$$
\left\|u C_{\varphi} f-u C_{\varphi} p_{n}\right\|_{\mathscr{B}} \leq\left\|u C_{\varphi}\right\|_{H(p, p, \phi) \rightarrow \mathscr{B}}\left\|f-p_{n}\right\|_{H(p, p, \phi)} \rightarrow 0
$$

as $n \rightarrow \infty$, since the operator $u C_{\varphi}: H(p, p, \phi) \rightarrow \mathscr{B}$ is bounded. Since $\mathscr{B}_{0}$ is a closed subset of $\mathscr{B}$, we obtain

$$
u C_{\varphi}(H(p, p, \phi)) \subset \mathscr{B}_{0} .
$$

Therefore $u C_{\varphi}: H(p, p, \phi) \rightarrow \mathscr{B}_{0}$ is bounded.

Theorem 4.5. Suppose that $\varphi$ is an analytic self-map of the unit disk, $u \in H(D), 0<$ $p<\infty$ and that $\phi$ is normal on $[0,1)$. Then, $u C_{\varphi}: H(p, p, \phi) \rightarrow \mathscr{B}_{0}$ is compact if and only if

$\lim _{|z| \rightarrow 1} \frac{\left(1-|z|^{2}\right)\left|u^{\prime}(z)\right|}{\phi(|\varphi(z)|)\left(1-|\varphi(z)|^{2}\right)^{1 / p}}=0 \quad$ and $\quad \lim _{|z| \rightarrow 1} \frac{\left(1-|z|^{2}\right)\left|u(z) \varphi^{\prime}(z)\right|}{\phi(|\varphi(z)|)\left(1-|\varphi(z)|^{2}\right)^{1+1 / p}}=0$. 
Proof. First, we assume that $u C_{\varphi}: H(p, p, \phi) \rightarrow \mathscr{B}_{0}$ is compact. Taking $f(z) \equiv 1$ we obtain that

$$
u \in \mathscr{B}_{0}
$$

From this, taking $f(z)=z$, and using the boundedness of $u C_{\varphi}: H(p, p, \phi) \rightarrow \mathscr{B}_{0}$ it follows that

$$
\lim _{|z| \rightarrow 1}(1-|z|)^{2}\left|u(z) \varphi^{\prime}(z)\right|=0
$$

Hence, if $\|\varphi\|_{\infty}<1$, from (30) and (31), we obtain that

$$
\lim _{|z| \rightarrow 1} \frac{\left(1-|z|^{2}\right)\left|u^{\prime}(z)\right|}{\phi(|\varphi(z)|)\left(1-|\varphi(z)|^{2}\right)^{1 / p}} \leq C \lim _{|z| \rightarrow 1} \frac{\left(1-|z|^{2}\right)\left|u^{\prime}(z)\right|}{\phi\left(\|\varphi\|_{\infty}\right)\left(1-\|\varphi\|_{\infty}^{2}\right)^{1 / p}}=0
$$

and

$$
\begin{aligned}
& \lim _{|z| \rightarrow 1} \frac{\left(1-|z|^{2}\right)\left|u(z) \varphi^{\prime}(z)\right|}{\phi(|\varphi(z)|)\left(1-|\varphi(z)|^{2}\right)^{1+1 / p}} \\
& \quad \leq C \lim _{|z| \rightarrow 1} \frac{\left(1-|z|^{2}\right)\left|u(z) \varphi^{\prime}(z)\right|}{\phi\left(\|\varphi\|_{\infty}\right)\left(1-\|\varphi\|_{\infty}^{2}\right)^{1+1 / p}}=0
\end{aligned}
$$

from which the conditions in (22) and (27) follow.

Hence, assume that $\|\varphi\|_{\infty}=1$. Let $\left(\varphi\left(z_{n}\right)\right)_{n \in \mathbb{N}}$ be a sequence such that $\lim _{n \rightarrow \infty}\left|\varphi\left(z_{n}\right)\right|$ $=1$. If necessary we can take a subsequence of $\left(\varphi\left(z_{n}\right)\right)_{n \in \mathbb{N}}$ (we use the same notation $\left.\left(\varphi\left(z_{n}\right)\right)_{n \in \mathbb{N}}\right)$. Set

$$
f_{n}(z)=\frac{\left(1-\left|\varphi\left(z_{n}\right)\right|^{2}\right)^{t+1}}{\phi\left(\left|\varphi\left(z_{n}\right)\right|\right)\left(1-\overline{\varphi\left(z_{n}\right)} z\right)^{1 / p+t+1}}, \quad n \in \mathbb{N}
$$

and

$$
g_{n}(z)=\frac{\left(1-\left|\varphi\left(z_{n}\right)\right|^{2}\right)^{t+2}}{\phi\left(\left|\varphi\left(z_{n}\right)\right|\right)\left(1-\overline{\varphi\left(z_{n}\right)} z\right)^{1 / p+t+2}}-\frac{\left(1-\left|\varphi\left(z_{n}\right)\right|^{2}\right)^{t+1}}{\phi\left(\left|\varphi\left(z_{n}\right)\right|\right)\left(1-\overline{\varphi\left(z_{n}\right)} z\right)^{1 / p+t+1}} .
$$

By the proof of Theorem 3.2 we know that

$$
\lim _{|\varphi(z)| \rightarrow 1} \frac{\left(1-|z|^{2}\right)\left|u(z) \varphi^{\prime}(z)\right|}{\phi(|\varphi(z)|)\left(1-|\varphi(z)|^{2}\right)^{1+1 / p}}=0
$$

and

$$
\lim _{|\varphi(z)| \rightarrow 1} \frac{\left(1-|z|^{2}\right)\left|u^{\prime}(z)\right|}{\phi(|\varphi(z)|)\left(1-|\varphi(z)|^{2}\right)^{1 / p}}=0 .
$$

Applying (30), (31), (32) and (33) with Lemmas 4.2 and 4.3 gives the desired result.

Conversely, from (7) we have that

$$
\begin{aligned}
& \left(1-|z|^{2}\right)\left|\left(u C_{\varphi} f\right)^{\prime}(z)\right| \\
& \quad \leq C\left(\frac{\left(1-|z|^{2}\right)\left|u^{\prime}(z)\right|}{\phi(|\varphi(z)|)\left(1-|\varphi(z)|^{2}\right)^{1 / p}}+\frac{\left(1-|z|^{2}\right)\left|u(z) \varphi^{\prime}(z)\right|}{\phi(|\varphi(z)|)\left(1-|\varphi(z)|^{2}\right)^{1+1 / p}}\right)\|f\|_{H(p, p, \phi)} .
\end{aligned}
$$


Taking the supremum in this inequality over all $f \in H(p, p, \phi)$ such that $\|f\|_{H(p, p, \phi)} \leq 1$, then letting $|z| \rightarrow 1$, we obtain that

$$
\lim _{|z| \rightarrow 1} \sup _{\|f\|_{H(p, p, \phi)} \leq 1}\left(1-|z|^{2}\right)\left|\left(u C_{\varphi}(f)\right)^{\prime}(z)\right|=0,
$$

from which by Lemma 4.1 we obtain that the operator $u C_{\varphi}: H(p, p, \phi) \rightarrow \mathscr{B}_{0}$ is compact.

From Theorems 4.4 and 4.5, we obtain the following corollaries:

\section{COROLLARY 4.6.}

Suppose that $\varphi$ is an analytic self-map of the unit disk, $0<p<\infty$ and that $\phi$ is normal on $[0,1)$. Then, the following statements hold.

(i) $C_{\varphi}: H(p, p, \phi) \rightarrow \mathscr{B}_{0}$ is bounded if and only if $C_{\varphi}: H(p, p, \phi) \rightarrow \mathscr{B}$ is bounded and $\varphi \in \mathscr{B}_{0}$.

(ii) $C_{\varphi}: H(p, p, \phi) \rightarrow \mathscr{B}_{0}$ is compact if and only if

$$
\lim _{|z| \rightarrow 1} \frac{\left(1-|z|^{2}\right)\left|\varphi^{\prime}(z)\right|}{\phi(|\varphi(z)|)\left(1-|\varphi(z)|^{2}\right)^{1+1 / p}}=0 .
$$

\section{COROLLARY 4.7.}

Suppose that $\varphi$ is an analytic self-map of the unit disk, $u \in H(D)$ and $0<p<\infty$. Then, the following statements hold.

(i) $u C_{\varphi}: A^{p} \rightarrow \mathscr{B}_{0}$ is bounded if and only if $u C_{\varphi}: A^{p} \rightarrow \mathscr{B}$ is bounded, $u \in \mathscr{B}_{0}$ and

$$
\lim _{|z| \rightarrow 1}\left(1-|z|^{2}\right)\left|u(z) \varphi^{\prime}(z)\right|=0 .
$$

(ii) $u C_{\varphi}: A^{p} \rightarrow \mathscr{B}_{0}$ is compact if and only if

$$
\lim _{|z| \rightarrow 1} \frac{\left(1-|z|^{2}\right)\left|u^{\prime}(z)\right|}{\left(1-|\varphi(z)|^{2}\right)^{2 / p}}=0 \quad \text { and } \quad \lim _{|z| \rightarrow 1} \frac{\left(1-|z|^{2}\right)}{\left(1-|\varphi(z)|^{2}\right)^{1+2 / p}}\left|u(z) \varphi^{\prime}(z)\right|=0 .
$$

\section{COROLLARY 4.8 .}

Suppose that $\varphi$ is an analytic self-map of the unit disk and $0<p<\infty$. Then, the following statements hold.

(i) $C_{\varphi}: A^{p} \rightarrow \mathscr{B}_{0}$ is bounded if and only if $C_{\varphi}: A^{p} \rightarrow \mathscr{B}$ is bounded and $\varphi \in \mathscr{B}_{0}$.

(ii) $C_{\varphi}: A^{p} \rightarrow \mathscr{B}_{0}$ is compact if and only if

$$
\lim _{|z| \rightarrow 1} \frac{\left(1-|z|^{2}\right)}{\left(1-|\varphi(z)|^{2}\right)^{1+2 / p}}\left|\varphi^{\prime}(z)\right|=0 .
$$

\section{Acknowledgement}

The first author of this paper is supported in part by the NNSF of China (No. 10671115), grants from specialized research fund for the doctoral program of higher education (No. 20060560002) and NSF of Guangdong Province (No. 06105648). 


\section{References}

[1] Cowen C C and MacCluer B D, Composition Operators on Spaces of Analytic Functions, Studies in Advanced Mathematics (Boca Raton: CRC Press) (1995)

[2] Hu Z J, Extended Cesàro operators on mixed norm spaces, Proc. Am. Math. Soc. 131(7) (2003) 2171-2179

[3] MacCluer B D and Zhao R, Essential norms of weighted composition operators between Bloch-type spaces, Rocky Mountain J. Math. 33(4) (2003) 1437-1458

[4] Madigan K and Matheson A, Compact composition operators on the Bloch space, Trans. Am. Math. Soc. 347(7) (1995) 2679-2687

[5] Ohno S, Weighted composition operators between $H^{\infty}$ and the Bloch space, Taiwan. J. Math. 5(3) (2001) 555-563

[6] Ohno S, Stroethoff K and Zhao R, Weighted composition operators between Bloch-type spaces, Rocky Mountain J. Math. 33 (2003) 191-215

[7] Ohno S and Zhao R, Weighted composition operators on the Bloch space, Bull. Austral. Math. Soc. 63 (2001) 177-185

[8] Rudin W, Function theory in the unit ball of $\mathbb{C}^{n}$ (New York: Springer Verlag) (1980)

[9] Shi J H and Luo L, Composition operators on the Bloch space, Acta Math. Sinica 16 (2000) 85-98

[10] Shields A L and Williams D L, Bounded projections, duality, and multipliers in spaces of analytic functions, Trans. Am. Math. Soc. 162 (1971) 287-302

[11] Stević S, On an integral operator on the unit ball in $\mathbb{C}^{n}$, J. Inequal. Appl. 1 (2005) 81-88

[12] Zhou Z H and Shi J H, Composition operators on the Bloch space in polydiscs, Complex Variables 46(1) (2001) 73-88

[13] Zhou Z, Composition operators between $p$-Bloch space and $q$-Bloch space in the unit ball, Progress in Natural Sci. 13(3) (2003) 233-236

[14] Zhu K, Operator Theory on Function Spaces, Marcel Dekker Inc. Pure and Applied Mathematics 139 (New York and Basel) (1990)

[15] Zhu K, Spaces of Holomorphic Functions in the Unit Ball (New York: Springer) (2005) 